\title{
EFFECT OF FISSION SOURCE SPECTRUM ON MONTE CARLO CALCULATION OF EX-CORE QUANTITIES
}

\author{
Eva E. Davidson ${ }^{1}$, Tara M. Pandya ${ }^{1}$, Katherine E. Royston ${ }^{1}$, Thomas M. Evans ${ }^{1}$, \\ Andrew T. Godfrey ${ }^{1}$, Shane C. Henderson ${ }^{1}$, Gary Wolfram ${ }^{2}$ and Joel M. Risner ${ }^{1}$ * \\ ${ }^{1}$ Oak Ridge National Laboratory \\ P.O. Box 5800, Oak Ridge, TN 37831-6170 \\ ${ }^{2}$ Pacific Northwest National Laboratory, TVA Headquarters \\ 1101 Market Street, Chattanooga, TN 32402
}

davidsonee@ornl.gov, pandyatm@ornl.gov, roystonke@ornl.gov, evanstm@ornl.gov, godfreyat@ornl.gov, hendersonsc@ornl.gov, gary.wolfram@pnnl.gov, risnerjm@ornl.gov

\begin{abstract}
The Consortium for Advanced Simulation of Light Water Reactors (CASL) Virtual Environment for Reactor Applications (VERA) offers unique capabilities to combine highfidelity in-core radiation transport with temperature feedback using MPACT and CTF with a follow-on fixed source transport calculation using the Shift Monte Carlo code to calculate ex-core quantities of interest. In these coupled calculations, MPACT provides a fission source to Shift for the follow-on radiation transport calculation. In past VERA releases, MPACT passed a spatially dependent source without the energy distribution to Shift. Shift then assumed a ${ }^{235} \mathrm{U}$ Watt spectrum to sample the neutron source energies. There were concerns that, in cases with burned or mixed oxide (MOX) fuel near the periphery of the core, the assumption of a ${ }^{235} \mathrm{U}$ Watt spectrum for the source neutron energies would not be accurate for studying ex-core quantities of interest, such as pressure vessel fluence or detector response. Therefore, two additional options were implemented in VERA for Shift to sample neutron source energies: (1) a nuclide-dependent Watt spectra for ${ }^{235} \mathrm{U},{ }^{238} \mathrm{U},{ }^{239} \mathrm{Pu}$, and ${ }^{241} \mathrm{Pu}$, and (2) to use the standard 51-energy group MPACT spectrum. Results show that the 51-group MPACT spectrum is not suitable for ex-core calculations because the groups have been fine-tuned for in-core calculations. Differences in relative detector response due to ${ }^{235} \mathrm{U}$ and nuclide-dependent Watt spectra sampling schemes were negligible; however, the use of nuclide-dependent Watt spectra for vessel fluence calculations was found to be important for fuel cycles with burned and fresh fuel.
\end{abstract}

KEYWORDS: ex-core analysis, hybrid methods, fission source spectrum, CASL

\footnotetext{
*Notice: This manuscript has been authored by UT-Battelle, LLC, under contract DE-AC05-00OR22725 with the US Department of Energy (DOE). The US government retains and the publisher, by accepting the article for publication, acknowledges that the US government retains a nonexclusive, paid-up, irrevocable, worldwide license to publish or reproduce the published form of this manuscript, or allow others to do so, for US government purposes. DOE will provide public access to these results of federally sponsored research in accordance with the DOE Public Access Plan (http://energy.gov/downloads/doe-public-access-plan).
} 


\section{INTRODUCTION}

The Consortium for Advanced Simulation of Light Water Reactors (CASL) Virtual Environment for Reactor Applications (VERA) [1] offers unique capabilities to combine high-fidelity in-core radiation transport with temperature feedback using the MPACT [2] deterministic solver and CTF [3] subchannel thermal hydraulics code with in-core and ex-core transport using the Shift [4] Monte Carlo (MC) code. Details about the full suite of ex-core capabilities in VERA can be found in an accompanying paper [5]. For the remainder of this paper, the focus is on the effect of the approximation used for the fission source spectra in Shift for the fixed-source MC calculation.

In these coupled calculations, MPACT provides a fission source to Shift for a follow-on fixed source radiation transport calculation to determine ex-core quantities of interest. In past releases of VERA, MPACT passed a spatially dependent source without the energy distribution to Shift. Shift then assumed a ${ }^{235} \mathrm{U}$ Watt spectrum to sample the neutron source energies. There were concerns that, in cases with burned fuel or mixed oxide (MOX) fuel near the core periphery, the assumption of a ${ }^{235} \mathrm{U}$ Watt spectrum for the neutron source energies would not be accurate for studying excore quantities of interest such as pressure vessel fluence or detector response. As a result, an investigation of the neutron source energy spectra used by Shift in VERA was undertaken.

Three options for the neutron source energy spectra in Shift were investigated: a ${ }^{235} \mathrm{U}$ Watt spectrum, nuclide-dependent Watt spectra based on ${ }^{235} \mathrm{U},{ }^{238} \mathrm{U},{ }^{239} \mathrm{Pu}$, and ${ }^{241} \mathrm{Pu}$, and the standard 51-energy group MPACT spectrum. The remainder of this paper is organized as follows. Section 2 presents the basic logic and implementation of using the different source spectra for ex-core calculations. Section 3 presents the findings from three different problems: (1) Shearon Harris detector response for varying moderator density, (2) Watts Bar Nuclear Plant Unit 1 (WBN1) vessel fluence, and (3) WBN1 reactor startup detector response. Problem (1) investigates using the three options for the neutron spectra whereas problems (2) and (3) investigate using the ${ }^{235} \mathrm{U}$ Watt spectrum and nuclide-dependent Watt spectra. Finally, Section 4 presents the overall conclusions about the effect of the fission source spectra on calculated ex-core quantities.

\section{METHODOLOGY}

As mentioned above, VERA performs high-fidelity ex-core calculations by taking advantage of its coupling to the Shift MC code; full details on these ex-core capabilities are given in an accompanying paper [5]. With this in-memory coupling, after MPACT and CTF complete a statepoint in-core transport calculation, the calculated fission source is passed to Shift to perform an MC fixed-source transport calculation on the full geometry to calculate the ex-core quantities of interest. In previously performed ex-core calculations, Shift only used the spatial distribution of this fission source (the total source strength from fission in neutrons per second in each pincell) passed from MPACT as given in Eq. 1:

$$
S_{c}=\sum_{i} \nu_{f, c}^{i} \Sigma_{f, c}^{i} \phi_{c} V_{c}
$$

where $i$ is a fissionable nuclide, $c$ is a pincell fuel region, $\nu_{f, c}^{i}$ is the number of neutrons produced from fission by nuclide $i, \Sigma_{f, c}^{i}$ is the macroscopic fission cross section of nuclide $i, \phi_{c}$ is the neutron flux in cell $c$ where the energy dependence has been omitted, and $V_{c}$ is the volume of cell $c$. 
The angular distribution of this fission source is always modeled as isotropic. This source in Shift was previously modeled as having only a ${ }^{235} \mathrm{U}$ Watt spectrum in energy. The Watt energy spectrum is represented in Eq. 2, where $C$ is a normalization factor and the constants ( $a$ and $b$ ) used in Shift are given in Table 1.

$$
W(E)=C e^{-E / a} \sinh \sqrt{b E}
$$

Table 1: Nuclide Watt Energy Spectra Constants Used in Shift

\begin{tabular}{llll}
\hline Nuclide & $\mathbf{a}(\mathrm{MeV})$ & $\mathbf{b}(1 / \mathrm{MeV})$ & Reference \\
\hline${ }^{235} \mathrm{U}$ & 0.96500 & 2.2900000 & MCNP5 manual (Cranberg spectrum) [6] \\
${ }^{238} \mathrm{U}$ & 0.88111 & 3.4005000 & MCNP5 manual [7] \\
${ }^{239} \mathrm{Pu}$ & 0.96600 & 2.8420000 & MCNP5 manual \\
${ }^{241} \mathrm{Pu}$ & 1.33196 & 0.0929657 & Manual fit to cross section data \\
\hline
\end{tabular}

To determine the effect of burned and MOX fuel on ex-core quantities, the ability to use the Watt spectra of ${ }^{235} \mathrm{U},{ }^{238} \mathrm{U},{ }^{239} \mathrm{Pu}$, and ${ }^{241} \mathrm{Pu}$ for the source neutrons was implemented in VERA. These four nuclides were chosen because they are the most prominent fission neutron producers in light water reactors (LWRs). For this option, MPACT sends the fission source strength from each of these four nuclides in each pincell to Shift after every statepoint. The source strength from all other nuclides are ignored since it is negligible for the LWR applications under analysis. This means, instead of the summation over all fissionable nuclides in Eq. 1, these four source strengths in each pincell are sent: $S_{c}^{235} U, S_{c}^{238} U, S_{c}^{239} P u$, and $S_{c}^{241} P u$. The constants used in Shift for these Watt spectra are given in Table 1. This work involved generating Watt spectrum constants for ${ }^{241} \mathrm{Pu}$, which was a simple fit of Eq. 2 to the tabulated data from the nuclear energy agency (NEA) JANIS 4.0 nuclear database. Finally, the ability to sample the neutron source energies based on the 51-energy group spectrum from MPACT was also added to VERA. With this option, MPACT sends Shift the full spatial and energy distribution for the fission source in each pincell.

\section{RESULTS}

Detector response and vessel fluence calculations were performed using the various neutron source energy sampling schemes with Shearon Harris and Watts Bar Unit 1 models in VERA. All Shift calculations were performed with Consistent Adjoint-Driven Importance Sampling (CADIS). The results from these calculations are discussed in detail in the next sections.

\subsection{Shearon Harris}

A study of the effect of these three different neutron source spectra sampling schemes (discussed in Section 2) on the power range detector relative ${ }^{10} \mathrm{~B}$ response was performed on the Shearon Harris (Harris) model, previously published in [8].

Figure 1a shows the in- and ex-core geometry. The in-core geometry was set up using VERA common input [9], and the ex-core geometry was modeled using General Geometry (GG) with a supplemental file. Two power range detectors are located within the bioshield in a wedge-shaped detector well. These detectors extend an equal distance above and below the active core mid-plane 
and contain two void regions: an inner void region enclosed in an aluminum tube, and an outer void region between the inner aluminum tube and an outer steel tube. The ${ }^{10} \mathrm{~B}$ detector response is calculated in the inner void region. The average ${ }^{10} \mathrm{~B}$ response in the two detector regions, top and bottom, is used to calculate the detector response relative to the first density point shown in Fig. 2.

Figure $1 \mathrm{~b}$ shows a radial slice of the reactor midplane for a modified Harris model in which the neutron pad is moved to a location at the 45-degree angle, in front of the detector. The geometry was modified to study whether the relative detector response would change as a result of a change in the geometry and the neutron source energy sampling scheme.

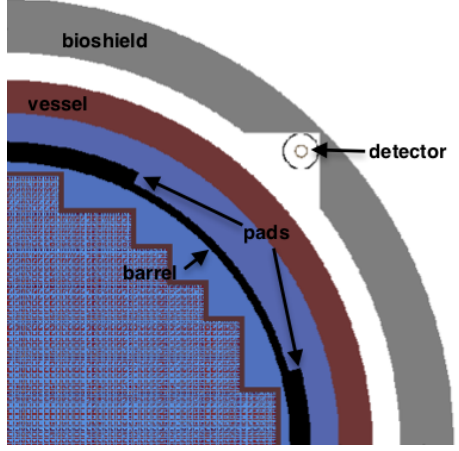

(a) Original

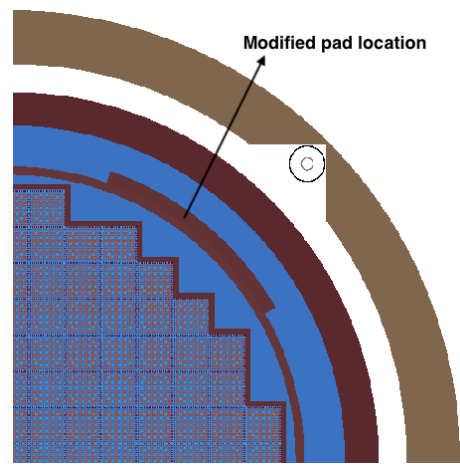

(b) Modified

Figure 1: Original and Modified Harris Quarter Core Geometry (X-Y Slice at Midplane)

The relative detector response was generated for the original Harris model using the three different neutron source energy sampling methods discussed in Section 2. This relative response is obtained by varying the moderator density in the downcomer region from 0.68 to $0.78 \mathrm{~g} / \mathrm{cc}$, and then dividing the absolute value of the detector response for each case by the first result obtained with a downcomer density of $0.68 \mathrm{~g} / \mathrm{cc}$. For the modified geometry, relative detector responses were generated assuming a ${ }^{235} \mathrm{U}$ Watt spectrum and the nuclide-dependent Watt spectra. MCNP results were also generated for the original Harris model shown in Fig. 1. The relative detector responses from these cases are all shown in Fig. 2, where the results generated with the modified geometry are denoted in the legend with Mod.Pad. Note that a comparison of the absolute values of the detector response from MCNP and Shift (standalone), generated with the original Harris geometry, were done previously in [8] and are not presented here. The absolute values from both the codes compared well giving further confidence in the validity of the relative detector response being calculated by Shift.

Figure 2 shows that the VERA results generated with the ${ }^{235} \mathrm{U}$ spectrum and nuclide-dependent Watt spectra sampling schemes are in good agreement with the MCNP results. However, the results generated with the 51-group MPACT spectrum are not in good agreement with the corresponding VERA or MCNP results. This was expected since this group structure was optimized for thermal neutrons in the core and not for ex-core calculations. The relative detector responses generated with the modified geometry where the pad is in-line with the detector are in good agreement with the corresponding results generated with the original geometry. The absolute value of the detector response is different from the original as a result of the change in the geometry, but the change in the relative detector response is negligible. Any effects from using a ${ }^{235} \mathrm{U}$ vs. a nuclide-dependent sampling scheme using the two Harris-based models are minimal, even though there is burned fuel 


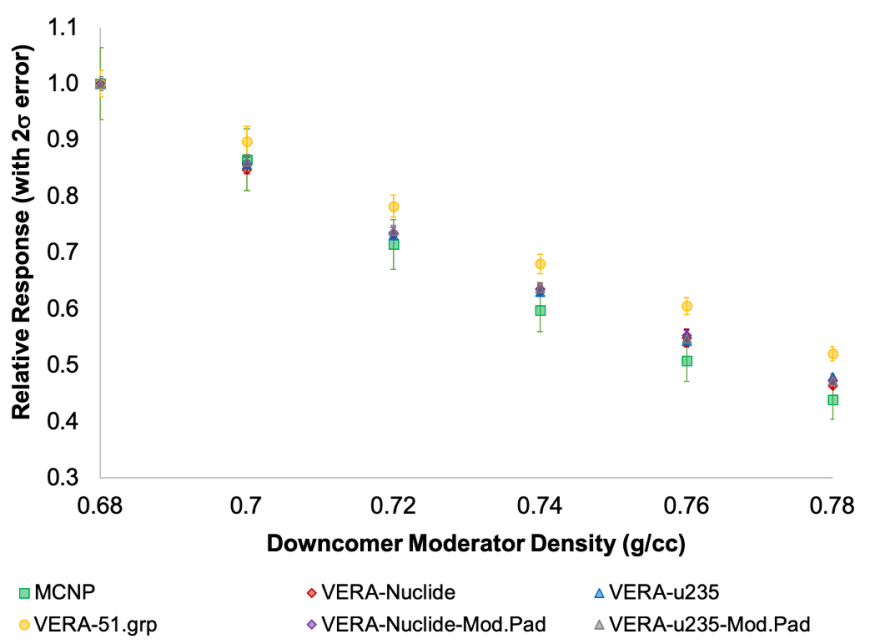

Figure 2: Harris Results with Different Fission Source Spectra

along the periphery of the core. A study for this work on the effect of the bioshield on the detector response showed that almost all ( 99\%) of the response is due to the neutrons scattering from the bioshield into the detector. Therefore, the insensitivity of the relative detector response to changes in the geometry and the neutron source energy sampling scheme are justified.

\subsection{Watts Bar Unit I}

A second study was performed using models of WBN1. A public Watts Bar (WB) model for Cycle 1 was used to analyze the difference in the vessel fluence results. In addition, the differences in the relative ${ }^{235} \mathrm{U}$ source range detector response generated with proprietary models of Cycles 8 and 15 during reactor startup were analyzed and compared to measured detector results. To perform these calculations, the boron concentration in parts per million (ppm) was set in the VERA input at 503 ppm to use for the Shift transport calculation. This was needed due to a limitation of VERA4.0 that has since been addressed. Note that the boron concentration required for refueling might be different and therefore, the ability to use the correct boron concentration in Shift at each state point will help alleviate making any approximations.

\subsubsection{Vessel fluence}

Vessel fluence calculations for WBN1 were performed using a public quarter-core model [10] for Cycle 1. This model does not include other ex-core features such as the bioshield or detectors; as shown in Fig. 3a. Figure 3b shows the maximum location for vessel fluence from neutrons with $\mathrm{E}>1 \mathrm{MeV}$. This maximum value is located at $\mathrm{z}=144.8 \mathrm{~cm}$ (approximately $55 \mathrm{~cm}$ below the core midplane) when using either the nuclide-dependent Watt spectra or the ${ }^{235} \mathrm{U}$ Watt spectrum for the neutron source energy in Shift. This is the axial location for the maximum fluence because the pin power peaking is the highest around this axial plane for more than half the cycle before a double-hump shaped profile forms and the axial pin power peaking shifts to the top. The maximum fluence values occur in different but adjacent theta bins for the two sampling schemes: theta $=0.761$ for nuclide-dependent Watt, and theta $=0.810$ for ${ }^{235} \mathrm{U}$ Watt. The maximum fluence calculated with 


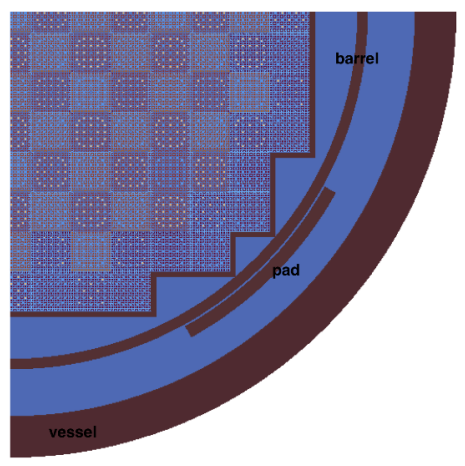

(a) $X$-Y Slice at Midplane $(\mathrm{z}=200 \mathrm{~cm})$

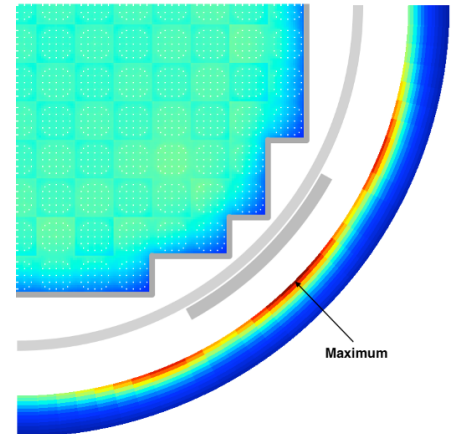

(b) Maximum Fluence Location

\section{Figure 3: WBN1 Vessel Fluence Calculation Using Nuclide-Dependent Watt Neutron Source}

the nuclide-dependent Watt spectra is $1.08 \times 10^{18} \mathrm{n} / \mathrm{cm}^{2}\left(0.1 \%\right.$ relative error), and the ${ }^{235} \mathrm{U}$ Watt spectrum is $1.05 \times 10^{18} \mathrm{n} / \mathrm{cm}^{2}(0.1 \%$ relative error $)$. This indicates a slight increase in the fluence calculated for $\mathrm{E}>1 \mathrm{MeV}$ when using the nuclide-dependent Watt neutron source energy sampling. Since WBN1 Cycle 1 consists of fresh fuel, the effects of the source spectra on the vessel fluence calculations are expected to be greater in cycles beyond the first in which burned fuel is loaded. These results indicate that it is important to use the more accurate nuclide-dependent Watt energy spectra for vessel fluence calculations.

\subsubsection{Startup}

Source Range Detectors (SRD) response calculations during reactor startup were previously performed for WB and compared with the measured relative detector response during startup for Cycles 8-15 [11]. There are two source range detectors: north and south. For these calculations, the SRD response is calculated in the north and south detectors as the core is systematically loaded with 1-10 assemblies before the startup of Cycles 8-15 in the presence of an antimonyberyllium secondary neutron source in the assemblies being loaded (more details in [11]). These SRD response measurements during startup measure the core reactivity and ensure the monitoring of inadvertent reactivity insertions. Previous calculations in [11] were run with the assumption that fission neutrons are born with a ${ }^{235} \mathrm{U}$ Watt spectrum. However, it is important to study any change to the relative detector response with the addition of the ability to sample using the nuclidedependent Watt spectra.

Figure 4 shows half of the WBN1 ex-core model used for the startup calculations, with annotations for various regions in the model. The SRDs are located at 0 (north) and 180 degrees (south), so these ex-core calculations did not use the quarter symmetry option in VERA.

The plots in Fig. 5 show the measured and calculated detector responses only in the south SRD during the startup of Cycles 8 and 15 (only 2 of the 8 cycles simulated). The differences seen between the relative detector responses calculated by VERA assuming a ${ }^{235} \mathrm{U}$ spectrum and nuclidedependent Watt spectra for neutron source energies are small; therefore, previous calculations performed assuming a ${ }^{235} \mathrm{U}$ Watt spectrum are still valid. Although the absolute value of the detector response does change with the source spectra used, the relative detector responses are in good 


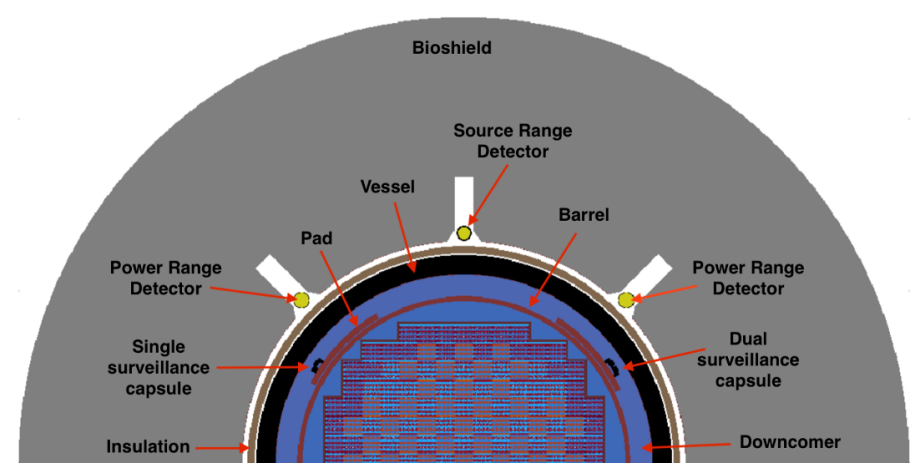

Figure 4: WB Full Core Geometry Y-Z Slice at Core Center

agreement, regardless of the neutron source energy spectra used. More details regarding these calculations will be given during the conference presentation.

\section{CONCLUSIONS}

For the study presented in this paper, a detailed analysis on the effect of the fission source energy spectra for ex-core detector calculations was performed using the Shearon Harris and WBN1 VERA models. The results show that for the ex-core detector responses, assuming that a neutron is born using the nuclide-dependent Watt spectra or the ${ }^{235} \mathrm{U}$ Watt spectrum does not produce noticeably different sets of results for the relative detector response. This is because the detector responses in the power range and SRDs are calculated as relative values and are mostly resulting from neutrons scattering from the bioshield into the detectors. VERA currently does not take into account the neutron emissions from Curium from burned fuel assemblies for the reactor startup calculations using secondary sources. This capability is currently being implemented in VERA to account for burned fuel emission sources. The work done for this study showed that using the 51-group MPACT spectrum produces the wrong relative detector response since this energy group structure has been fine-tuned for in-core and not ex-core calculations. Finally, it is expected that the nuclide-dependent Watt spectra will provide more accurate results when performing vessel fluence calculations with VERA because the spectral effects are more significant for these calculations with mixed burned and fresh fuel, especially when considering the inner vessel surface. Therefore,

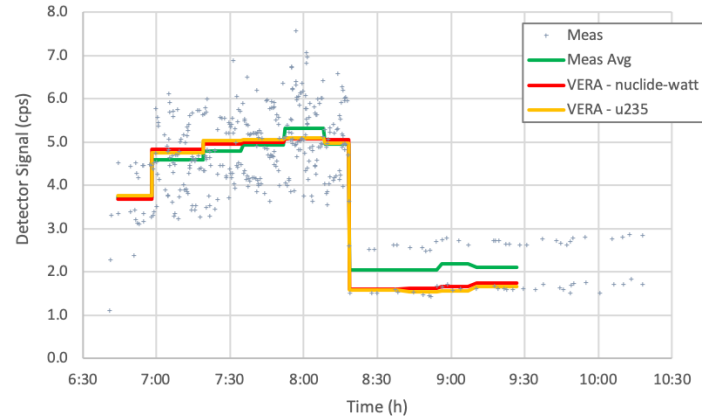

(a) Cycle 8 South Detector

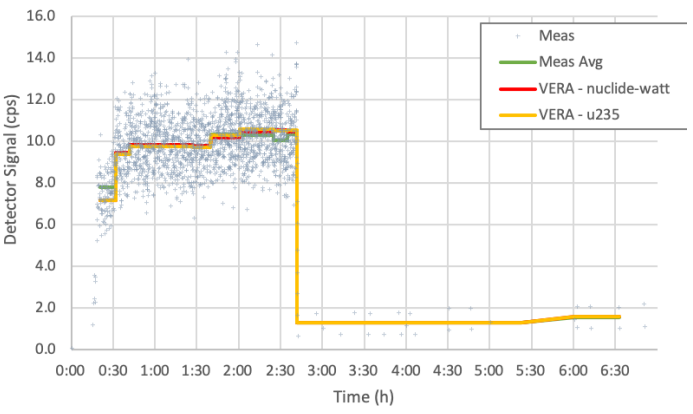

(b) Cycle 15 South Detector

Figure 5: Measured and Calculated SRD Responses for WBN1 Startup 
it is important to account for the slightly higher energy neutrons from ${ }^{239} \mathrm{Pu}$ fissions in burned fuel along the periphery of the core.

\section{ACKNOWLEDGMENTS}

This research was supported by the Consortium for Advanced Simulation of Light Water Reactors (http://www.casl.gov), an Energy Innovation Hub (http://www.energy.gov/hubs) for Modeling and Simulation of Nuclear Reactors under US Department of Energy (DOE) Contract No. DE-AC0500OR22725. This research also used resources of the Compute and Data Environment for Science (CADES) at ORNL, which is supported by the Office of Science of the US DOE.

\section{REFERENCES}

[1] J. A. Turner, K. Clarno, M. Sieger, R. Bartlett, B. Collins, R. Pawlowski, R. Schmidt, and R. Summers. "The Virtual Environment for Reactor Applications (VERA): Design and architecture." Journal of Computational Physics, volume 326, pp. 544-568 (2016).

[2] B. Collins, S. Stimpson, B. Kelley, M. Young, B. Kochunas, A. Graham, E. Larsen, T. Downar, and A. Godfrey. "Stability and accurace of 3D neutron transport simulation using the 2D/1D method in MPACT." Journal of Computational Physics, volume 326, pp. 612-628 (2016).

[3] M. N. Avramova. "CTF: A Thermal Hydraulic Sub-Channel Code for LWR Transient Analyses, Users Manual.” Technical report, Pennsylvania State University Department of Nuclear Engineering (2009).

[4] T. M. Pandya, S. R. Johnson, T. M. Evans, G. G. Davidson, S. P. Hamilton, and A. T. Godfrey. "Implementation, capabilities, and benchmarking of Shift, a massively parallel Monte Carlo radiation transport code." Journal of Computational Physics, volume 308, pp. 239-272 (2016).

[5] T. M. Pandya, K. E. Royston, T. M. Evans, A. T. Godfrey, E. E. Davidson, and S. C. Henderson. "High-Fidelity Ex-core Capabilities in VERA." In Proceedings of the PHYSOR 2020. American Nuclear Society (2020).

[6] X-5 Monte Carlo Team. "MCNP A General N-Particle Transport Code, Version 5, Volume II.” Technical Report LA-CP-03-0245, Los Alamos National Laboratory (2003).

[7] X-5 Monte Carlo Team. "MCNP A General N-Particle Transport Code, Version 5, Volume III.” Technical Report LA-CP-03-0284, Los Alamos National Laboratory (2003).

[8] H. P. Smith, E. E. Davidson, A. T. Godfrey, and T. M. Pandya. "An Analysis of Various Solution Strategies and Perturbations on Inputs of the Reactor Shielding Problem." In $20^{\text {th }}$ Topical Meeting of the Radiation Protection Shielding Division. American Nuclear Society (2018).

[9] S. Palmtag and A. T. Godfrey. "VERA Common Input User Manual." Technical Report CASL-U-2014-0014-002, CASL (2015).

[10] A. T. Godfrey. "VERA Core Physics Benchmark Progression Problem Specifications." Technical Report CASL-U-2012-0131-004, CASL (2014).

[11] C. Gentry, A. Godfrey, E. Davidson, G. Ilas, B. Collins, S. Hart, K. Kim, T. Pandya, K. Royston, G. Davidson, S. Johnson, T. Evans, G. Wolfram, and S. Palmtag. "Source Range Detector Response Modeling Using VERA." In Proceedings of GLOBAL/Top Fuel 2019. American Nuclear Society (2019). 